The usefulness of higher-order constitutive relations for describing the Knudsen layer

Duncan A. LockerbyJason M. ReeseMichael A. Gallis

Citation: Physics of Fluids 17, 100609 (2005); doi: 10.1063/1.1897005

View online: http://dx.doi.org/10.1063/1.1897005

View Table of Contents: http://aip.scitation.org/toc/phf/17/10

Published by the American Institute of Physics

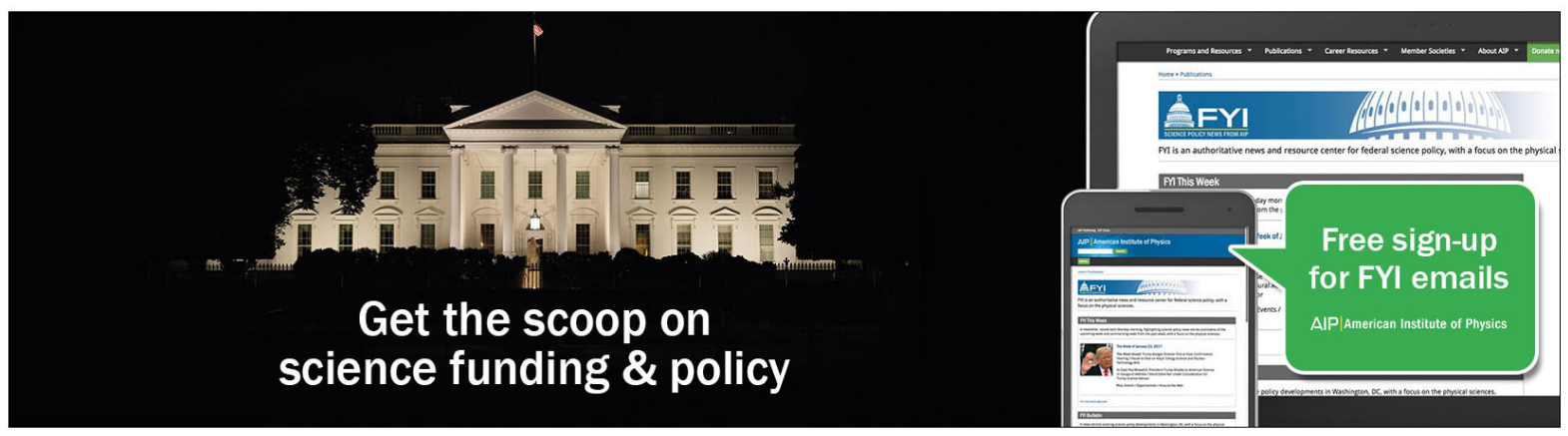




\title{
The usefulness of higher-order constitutive relations for describing the Knudsen layer
}

\author{
Duncan A. Lockerby \\ School of Engineering and Design, Brunel University, Uxbridge UB8 3PH, United Kingdom \\ Jason M. Reese \\ Department of Mechanical Engineering, University of Strathclyde, Glasgow G1 1XJ, United Kingdom \\ Michael A. Gallis \\ Engineering Sciences Center, Sandia National Laboratories, P.O. Box 5800, Albuquerque, \\ New Mexico 87185-0826
}

(Received 10 November 2004; accepted 11 February 2005; published online 3 October 2005; publisher error corrected 13 October 2005)

\begin{abstract}
The Knudsen layer is an important rarefaction phenomenon in gas flows in and around microdevices. Its accurate and efficient modeling is of critical importance in the design of such systems and in predicting their performance. In this paper we investigate the potential that higher-order continuum equations may have to model the Knudsen layer, and compare their predictions to high-accuracy DSMC (direct simulation Monte Carlo) data, as well as a standard result from kinetic theory. We find that, for a benchmark case, the most common higher-order continuum equation sets (Grad's 13 moment, Burnett, and super-Burnett equations) cannot capture the Knudsen layer. Variants of these equation families have, however, been proposed and some of them can qualitatively describe the Knudsen layer structure. To make quantitative comparisons, we obtain additional boundary conditions (needed for unique solutions to the higher-order equations) from kinetic theory. However, we find the quantitative agreement with kinetic theory and DSMC data is only slight. (C) 2005 American Institute of Physics. [DOI: 10.1063/1.1897005]
\end{abstract}

\section{INTRODUCTION}

In the region of a gas flow very close to a solid surface, conventional fluid dynamic models are not appropriate. This is because there are insufficient molecular-molecular and molecular-surface collisions over this very small scale to justify the assumption of quasi thermodynamic-equilibrium - an assumption upon which the Navier-Stokes equations, and the associated no-slip boundary condition, depend (see Ref. 1 for a good discussion of this subject). Two defining characteristics of this near-surface region of a gas flow are the following: first, there is a finite velocity of the gas at the surface (velocity slip), and second, there exists a nonNewtonian stress/strain-rate relationship that extends a few molecular mean free paths into the gas (known as the Knudsen layer or kinetic boundary layer). Although the Knudsen layer has thermal aspects, the scope of this paper is restricted to incompressible, slightly rarefied, isothermal gas flows, where thermal phenomena are of lesser importance. For brevity, this general class of flow will be referred to here as "low-speed gas microflows."

Ordinarily, the Knudsen layer and velocity slip are negligible in comparison to macroscopic features of the flow. However, in rarefied conditions these effects become an important part of the overall flow field. Broadly, the degree of gas rarefaction can be expressed by the dimensionless Knudsen number,

$$
\mathrm{Kn}=\frac{\lambda}{L},
$$

where $\lambda$ is the molecular mean free path and $L$ is a length scale that is characteristic of some global feature of the flow. For planar Poiseuille flow (pressure-driven flow in a channel), with a Knudsen number of $\mathrm{Kn}=0.05$ (based on a length scale equal to half the channel height), the mass flow rate is around $15 \%$ greater than it would be in nonrarefied conditions (see the calculation in Appendix A). Approximately $70 \%$ of this mass flow rate increase is due to the velocity slip at the wall, and $30 \%$ due to the non-Newtonian structure of the Knudsen layer. Contrary to what is often assumed, therefore, the Knudsen layer has a significant impact even at relatively low Knudsen numbers, and so is an essential consideration when modeling low-speed gas microflows.

While the Knudsen layer has been investigated extensively using kinetic theory, ${ }^{2-4}$ the ability to capture it within a continuum-fluid formulation suitable for current computational fluid dynamics (CFD) techniques would have distinct advantages in terms of computational efficiency. Although slip can be incorporated using velocity boundary conditions, the Navier-Stokes constitutive relations cannot model the nonlinear stress/strain-rate behavior within the Knudsen layer. A remedy to this shortcoming of conventional CFD may be the use of higher-order constitutive relations. In this paper we evaluate and compare the ability of different sets of available constitutive relations to improve the continuum- 


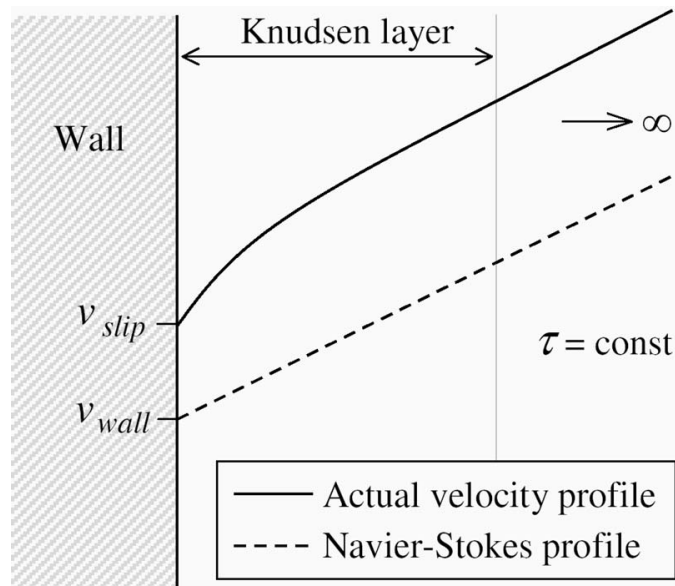

FIG. 1. Schematic of Kramer's problem: a gas in a half space, bounded by a planar wall, subject to a uniform and constant shear stress $\tau$. The NavierStokes solution with no-slip boundary condition $(--)$ departs significantly from the actual velocity profile (-), and the velocity of the gas at the wall $\left(\nu_{\text {slip }}\right)$ is different to the velocity of the wall itself $\left(\nu_{\text {wall }}\right)$.

fluid predictions of low-speed gas microflows; specifically, their ability to model the Knudsen layer.

\section{DISCRETE-MOLECULAR APPROACHES}

A gas flow generated by a uniformly applied shear stress and bounded by one parallel surface is a configuration known as Kramer's problem (see Fig. 1). Kramer's problem allows us to investigate the one-dimensional isothermal Knudsen layer in isolation. It is, therefore, the most fundamental benchmark case for testing methods of modeling the Knudsen layer. And so, one might argue, if a continuum model cannot accurately solve Kramer's problem, it will probably not be a good model for low-speed gas microflows in general.

In this section, and for later comparison with continuumfluid models, we describe solutions to Kramer's problem using discrete-molecular approaches. The data denoted by circles in Fig. 2 have been obtained using the direct simulation Monte Carlo (DSMC) method; a statistical molecular dynamics approach that tracks the motion and collisions of a large number of representative particles. The DSMC code we have used is a modified version of DSMC1 published by Bird. ${ }^{5}$ In this case, our solution to Kramer's problem is a simulation of low-speed (Mach number $=0.05$ ) Couette flow of argon gas (Kramer's problem is the limiting case of Couette flow as $\mathrm{Kn} \rightarrow 0$ ). In our Couette flow simulations the height of the channel is roughly $8 \lambda$ (for $\mathrm{Kn}=0.125$ ), which is amply sufficient to accommodate the Knudsen layers on both walls without interference with each other. More than 10 $\times 10^{9}$ molecular moves have been performed computationally, and the statistical variation in our calculated stress field is three orders of magnitude less than the actual value. The calculation was performed on 1000 processors of ASCI Red, a 3 TFLOP capable massively parallel computer; full simulation details are given in Table $\mathrm{I}$.

The solid line in Fig. 2 is a solution of the linearized Boltzmann equation obtained using the Bhatnagar-GrossKrook (BGK) approximation; ${ }^{3}$ for the low-speeds consid-

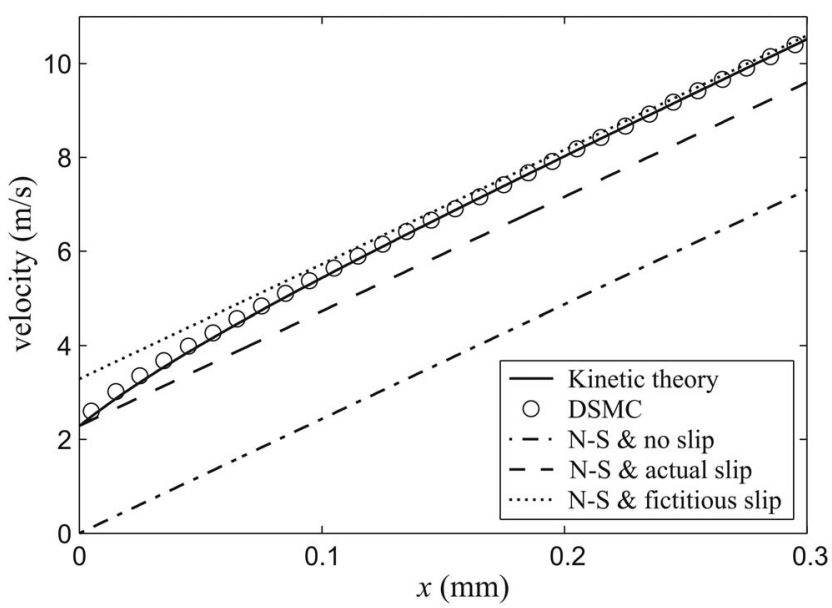

FIG. 2. Velocity profiles in Kramer's problem (planar wall at $x=0$ ). DSMC results for argon (o); kinetic theory (-); Navier-Stokes with fictitious slip $(\ldots)$; Navier-Stokes with actual slip $(--)$; Navier-Stokes with no slip $(-\cdot-)$. Values extracted from DSMC data: applied shear stress $\tau=0.5 \mathrm{~N} \mathrm{~m}^{-2}$; dynamic viscosity $\mu=20.5 \times 10^{-6} \mathrm{~N} \mathrm{~m}^{-2} \mathrm{~s}^{-1}$; mean free path $\lambda=118 \times 10^{-6} \mathrm{~m}$. For the equivalent DSMC calculation of Couette flow, the second wall is situated at $x=1 \mathrm{~mm}$.

ered, this approximate method is a fair one. It can be seen that the discrete-molecular solutions and kinetic theory are in good agreement with each other, and so, in the absence of reliable experimental data, can collectively be considered as a suitable benchmark solution for comparison with our continuum-fluid solutions.

\section{NAVIER-STOKES SOLUTIONS}

The dash-dot line in Fig. 2 indicates the prediction of the Navier-Stokes equations with the conventional no-slip boundary condition. The dashed line is the prediction obtained when using a velocity boundary condition taken from the kinetic theory solution. The viscosity and shear stress are constant throughout the Knudsen layer: the values of these, which are used in all of the continuum calculations in this paper, have been obtained directly from our DSMC calculation.

Commonly, "fictitious" (or "macro") slip boundary conditions are used in conjunction with the Navier-Stokes solution so that good agreement can, at least, be obtained outside of the Knudsen layer (the dotted line in Fig. 2). However, the disadvantage of this approach is the need for some part of the domain near to the wall to be fictitious. At higher Knudsen numbers (although still significantly less than 1) the proportion of the domain that is therefore represented fictitiously is substantial. Knudsen-layer corrections based on numerical calculations in kinetic theory can remove this fictitious element, ${ }^{4}$ but this adds substantial complication to an otherwise simple continuum calculation. What would be more desirable, from engineering and computational viewpoints, is to model the physics of the Knudsen layer using a single continuum-fluid formulation with no additional input. We will now examine whether higher-order continuum-fluid equations may be able to fulfill this role. 
TABLE I. DSMC parameters used in our simulation of low-speed Couette flow of argon.

\begin{tabular}{ll}
\hline \hline \multicolumn{1}{c}{ Simulation parameter } & \multicolumn{1}{c}{ Value } \\
\hline Wall separation & $1 \mathrm{~mm}$ \\
Difference in wall velocities & $30.8 \mathrm{~m} / \mathrm{s}$ \\
Wall surface temperature & $273.2 \mathrm{~K}$ \\
Domain size & $0.5 \mathrm{~mm}$ (half channel) \\
Grid points & 50 \\
Number of particles & $1.5 \times 10^{6}$ \\
Calculated particle moves & $13.65 \times 10^{9}$ \\
Pressure & $52 \mathrm{~Pa}$ \\
Viscosity & $2.117 \times 10^{-5}$ Pa s \\
Temperature exponent of viscosity coefficient $(\omega)$ & 0.81 \\
Exponent in variable soft sphere (VSS) molecular model $(\alpha)$ & 1.4 \\
Properties of molecular reflection at wall & Pure Maxwellian diffuse reflection \\
\hline
\end{tabular}

\section{HIGHER-ORDER CONTINUUM-FLUID EQUATIONS}

Numerous higher-order continuum-fluid equation sets have been proposed, by various researchers, for flows that depart from quasi thermodynamic-equilibrium. These include Burnett, ${ }^{6}$ super-Burnett, ${ }^{7}$ Grad's 13 moment, ${ }^{8}$ Eu's generalized hydrodynamics $(\mathrm{GH}),{ }^{9}$ Zhong's augmented Burnett, ${ }^{10}$ Lumpkin's reduced Burnett, ${ }^{11}$ BGK-Burnett, ${ }^{12}$ regularized Burnett, ${ }^{13} \mathrm{R} 13,{ }^{14}$ and Woods ${ }^{15}$ equations. This is not an exhaustive list, but includes the most commonly used and cited. (Unfortunately, lack of space precludes a detailed description and full evaluation of their relative merits here.)

There are three basic reasons why there are so many competing sets of different higher-order equations. First, constitutive relations of higher-order than the Navier-Stokes equations have demonstrated potential in modeling rarefied hypersonic flows, specifically, in the prediction of onedimensional shock wave thickness. This success has generated significant research interest. Second, these higher-order equations are all plagued by numerical and physical instabilities and, in many cases, produce nonphysical flow predictions. This has prompted the development of a number of variant forms of the two main equation families (i.e., Burnett's and Grad's) in an attempt to eliminate these flaws. Third, no single equation set has demonstrated universal superiority in the prediction of rarefied gas flows-it is an active research question as to which is the "best" set of equations.

All of the higher-order continuum equations we consider in this paper originate from, or are based on, either the Chapman-Enskog series solution technique (which generates the Burnett equations) or Grad's 13 moment approach. Surprisingly, after performing the linearization (see Appendix B) and one dimensionalization appropriate for Kramer's problem, the Burnett and Grad equations both reduce to the Navier-Stokes equations-the dashed line in Fig. 2: i.e., neither can predict a Knudsen layer. This nonresult is partially supported by Kogan, ${ }^{2}$ who demonstrated that the ChapmanEnskog series (which has similarities to Grad's approach) does not provide a solution to the Boltzmann equation in the Knudsen layer. Eu's generalized hydrodynamics, Woods' frame-indifferent equations and Lumpkin's reduced Burnett are other higher-order equation sets that reduce to the Navier-Stokes equations for Kramer's problem.

Examining some of the other proposed sets of equations: for the linearized one-dimensional form of Zhong's augmented Burnett and BGK-Burnett equations, the expression for the shear stress $\tau$ (which is constant in Kramer's problem) is of the general form

$$
\tau=-\mu \frac{d \nu}{d x}+A \frac{\mu^{3}}{p \rho} \frac{d^{3} \nu}{d x^{3}},
$$

where $\nu$ is the velocity parallel to the surface, $x$ is in a direction perpendicular to the surface, $\mu$ is the gas viscosity, $p$ is the pressure, and $\rho$ is the density. For the BGK-Burnett equations, $A=1$, and for Zhong's augmented Burnett equations, $A=1 / 6$. For the Burnett equations, $A=0$. Notice that the first term in Eq. (2) is equivalent to the Navier-Stokes shear stress; one of the desirable features of higher-order continuum equations is that they reduce to the Navier-Stokes equations in any nonrarefied regions of the gas flow.

For the linearized one-dimensional super-Burnett equations the shear stress expression has a slightly different form (the sign of the higher-order term is inverted),

$$
\tau=-\mu \frac{d \nu}{d x}-\frac{2}{3} \frac{\mu^{3}}{p \rho} \frac{d^{3} \nu}{d x^{3}} .
$$

As will be demonstrated later this has a significant impact on the solution.

In the regularized Burnett and R13 equations, the shear stress is not expressed explicitly, as above. Instead, the flow velocity is calculated by solving the following equations simultaneously:

$$
\tau=-\mu \frac{d \nu}{d x}-\frac{2}{5} \frac{\mu}{p} \frac{d q}{d x},
$$

and

$$
q=B \frac{\mu^{2}}{p \rho} \frac{d^{2} q}{d x^{2}},
$$

where $q$ is the heat flux parallel to the surface and $B=9 / 5$ for the R13 equations. For Grad's and Eu's equations, $B=0$. The 
TABLE II. Values for Knudsen layer thickness in Kramer's problem, predicted by various higher-order continuum equations and a solution to the linearized Boltzmann equation.

\begin{tabular}{cc}
\hline \hline Solution method & Knudsen layer thickness \\
\hline Burnett (Reference 6), Super-Burnett (Reference 7), Grad's 13 moment (Reference 8), & No Knudsen layer predicted \\
Eu's GH (Reference 9), Lumpkin's reduced Burnett (Reference 11), Woods (Reference 15) & $0.9 \lambda$ \\
Zhong's augmented Burnett (Reference 10) & $1.4 \lambda$ \\
Linearized Boltzmann equation (Reference 3)(kinetic theory) & $2.1 \lambda$ \\
BGK-Burnett (Reference 12) & $2.8 \lambda$ \\
R13 (Reference 14) & $4.9 \lambda$ \\
Regularized Burnett (Reference 13) & \\
\hline \hline
\end{tabular}

coefficients in the regularized Burnett equations are not known $a$ priori, and here we use a value suggested in Ref. 14: $B=27 / 5$. This value is obtained by comparing the regularized Burnett with the R13 equations for the onedimensional case where flow velocity is in the same direction as the flow variation (such as in a one-dimensional shock wave). Interestingly, both the R13 and regularized Burnett equations can predict a heat flux in the absence of a temperature gradient.

\section{A. Boundary conditions}

In order to test the predictions of the higher-order continuum equations (2)-(5) we need to provide new boundary conditions to obtain a unique solution. In most flows of interest there is no indication as to what these additional boundary conditions might be. For the purposes of testing the equation sets on this benchmark Kramer's problem, however, we can use boundary conditions extracted from the linearized solution of the Boltzmann equation (the solid line in Fig. 2).

The first boundary condition we obtain (which is required even for the Navier-Stokes solution) is the velocity slip due to diffuse molecular reflection at the wall,

$$
\nu_{x=0}=-\lambda \frac{\tau}{\mu} \sqrt{\frac{2}{\pi}}
$$

where the definition used here for the mean free path is

$$
\lambda=\mu \sqrt{\frac{\pi}{2 p \rho}} .
$$

Second, as $x$ tends to infinity (i.e., outside of the Knudsen layer) the shear stress is related to the rate of strain by the Navier-Stokes constitutive relation:

$$
\left.\frac{d \nu}{d x}\right|_{x \rightarrow \infty}=-\frac{\tau}{\mu}
$$

The final boundary condition that is required to produce a unique solution to Eqs. (2)-(5), we obtain by evaluating the rate of strain at the wall:

$$
\left.\frac{d \nu}{d x}\right|_{x=0}=-1.7 \frac{\tau}{\mu} .
$$

This value is taken from a curve-fitted approximation to Cercignani's solution of Kramer's problem (see Ref. 16 for details).

\section{B. Super-Burnett solution}

The velocity profile predicted by the super-Burnett equations can be obtained by solving the ordinary differential equation (3):

$$
\nu=k_{1}+\frac{\tau}{\mu} x+k_{2} \cos \left(k_{3} x\right)+k_{4} \sin \left(k_{3} x\right),
$$

where $k_{1-4}$ are constants. Equation (10) is oscillatory in space, but since the strain-rate tends to a constant value away from the surface—enforced by boundary condition (8) - the oscillatory part must be zero, i.e.,

$$
k_{2}=k_{4}=0 \text {. }
$$

The resulting solution is linear and, again, equivalent to that produced by the Navier-Stokes equations (the dashed line in Fig. 2).

\section{Knudsen layer solutions}

The remaining equation sets, Eqs. (2), (4), and (5), can be solved analytically, and all have solutions of the form:

$$
\nu=k_{1}-\frac{\tau}{\mu} x+k_{2} e^{ \pm k_{3} x} .
$$

Together with the boundary conditions of Eqs. (6), (8), and (9), this provides the following unique solution for the velocity profile:

$$
\nu=-\frac{\tau}{\mu}\left[x+\lambda \sqrt{\frac{2}{\pi}}+\frac{7}{10 C} \lambda\left(1-e^{-C x / \lambda}\right)\right] .
$$

For the BGK-Burnett equations, $C=\sqrt{\pi / 2}$; for the regularized Burnett equations, $C=\sqrt{5 \pi / 54}$; for Zhong's augmented Burnett equations, $C=\sqrt{3} \pi$; and for the R13 equations, $C$ $=\sqrt{5 \pi / 18}$.

The velocity profile described by Eq. (13) appears to have a Knudsen-layer-like form. For each equation set, the thickness of this Knudsen layer is of the order of a few mean 


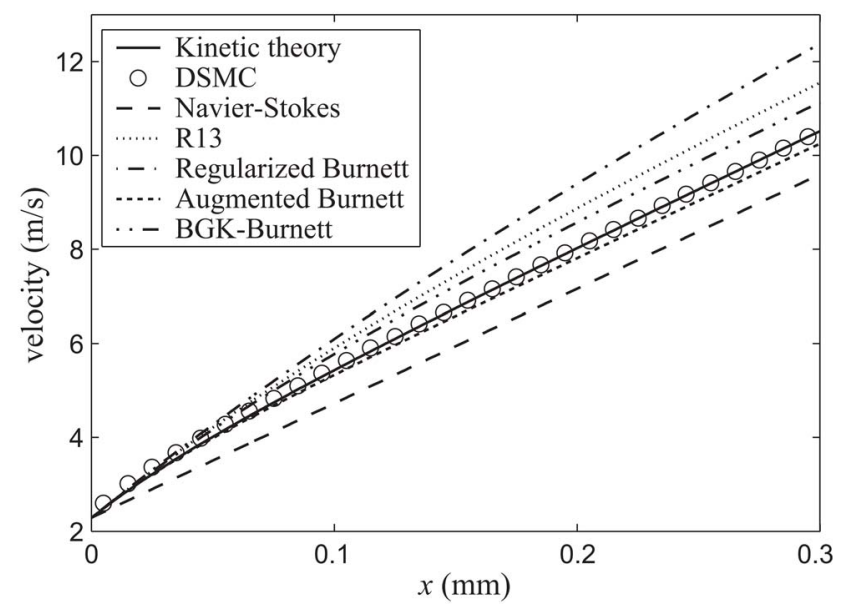

FIG. 3. Velocity profiles in Kramer's problem (planar wall at $x=0$ ). DSMC results for argon (o); kinetic theory (-); Navier-Stokes, Burnett, superBurnett, Grad's 13 moment $\left(-{ }_{-}\right)$; R13 (..); regularized Burnett (- .); Zhong's augmented Burnett (---); BGK-Burnett (- -.). Details of the flow as given under Fig. 2.

free paths. Qualitatively this is reasonable. Quantitatively, however, the agreement with the kinetic theory solution and DSMC data is not particularly good. A comparison is shown in Table II of the Knudsen layer thickness (here defined as the distance from the wall at which the strain rate reaches within $5 \%$ of the Navier-Stokes value). The thickness calculated by kinetic theory is $1.4 \lambda$, and that by the various higher-order continuum equations is in the range $0.9 \lambda-4.9 \lambda$.

Figure 3 shows a comparison of the velocity profiles predicted by Eq. (13) for the various higher-order formulations and the DSMC and kinetic theory solutions. As a reference, the Navier-Stokes prediction is also plotted (and is equivalent to the Burnett, super-Burnett, Grad's 13 Moment, Eu's GH, Lumpkin's reduced Burnett, and Woods solutions). There is a significant spread in the continuum equations' predictions; the closest match to the discrete-molecular data is provided by Zhong's augmented Burnett equations.

\section{DISCUSSION AND SUMMARY}

Care must be taken in drawing appropriate conclusions from these results. It would be unjustified to dismiss or advocate a particular equation set based on a single test case. However, since the Knudsen layer is such an important feature of gas microflows, the inability of any model to accurately capture the Knudsen layer in its simplest form might point to a general problem in that set of model equations for low-speed microfluidics.

In this paper we have compared high-accuracy DSMC calculations with a standard kinetic theory solution. The agreement between these two approaches is good, and so these serve as a benchmark solution for the form of the Knudsen layer. Neither the Burnett, super-Burnett, or Grad's 13 moment equations can model this Knudsen layer, which then raises the question of whether variant forms of these basic equation families are likely to be able to demonstrate any better predictive capabilities. However, qualitatively accurate Knudsen layer predictions can be obtained with a number of the higher-order continuum equations that we have considered. Zhong's augmented Burnett equations provide the closest agreement with the DMSC data and kinetic theory, but, in part, this must be coincidental since the augmentations to the original Burnett equations were chosen in an ad hoc way to ensure stability and not to expand the physical model. ${ }^{10}$ On the whole, the ability of the higherorder equations to quantitatively match the DSMC data is poor.

Given the difficulty in ascertaining the additional boundary conditions required to generate unique solutions to any set of higher-order governing equations, it may prove more practical to incorporate, rather than directly model, Knudsen layer effects within conventional continuum-fluid methods. As mentioned above, this could be done either by using precalculated results from kinetic theory to correct NavierStokes solutions obtained with fictitious slip boundary conditions, ${ }^{4}$ or by capturing the Knudsen layer structure within a Navier-Stokes solution by scaling the stress/strainrate relationship using a "wall-function" approach. ${ }^{16}$

The findings of this study are not directly applicable to high-speed or nonisothermal flows, and we cannot therefore comment on the usefulness of higher-order continuum equations for modeling such situations. For incompressible isothermal microflows, however, we have shown that the Knudsen layer, which is a dominant rarefaction effect in these cases, cannot be modeled accurately by the majority of available higher-order continuum equations. This does, at least, raise questions about the usefulness of these models for lowspeed gas microflows in general.

\section{ACKNOWLEDGMENTS}

The authors thank the referees of this paper for their helpful comments. D.A.L. and J.M.R. are grateful to the UK's Leverhulme Trust (Research Project Grant No. F/07040/G) for supporting this research financially. M.A.G. performed his work at Sandia National Laboratories. Sandia is a multiprogram laboratory operated by Sandia Corporation, a Lockheed Martin Company, for the United States Department of Energy's National Nuclear Security Administration under Contract No. DE-AC04-94AL85000.

\section{APPENDIX A}

For low-speed, low-Kn, isothermal planar Poiseuille flow, the velocity slip (with diffuse molecular reflection at the wall) and Knudsen layer effects can both be accounted for within a Navier-Stokes solution by using "fictitious" (sometimes called "macro") slip boundary conditions. Cercignani obtained such conditions from a linearized solution of the Boltzmann equation for Kramer's problem: ${ }^{3}$

$$
\nu_{\text {slip }}=A \lambda \frac{d \nu}{d x}
$$

where $\nu$ is the velocity parallel to the wall, $x$ is the direction normal to the wall, and the macro slip coefficient is $A$ $=1.146$. At low Knudsen numbers, the difference between the actual velocity profile and the one generated by the Navier-Stokes equations with boundary condition (A1) is 
small and, for these rough estimations, acceptable. This theory also assumes that the stress is constant through the Knudsen layer, which for Posieuille flow at $\mathrm{Kn}=0.05$ (based on half-channel height) is a fair, if not precise, approximation (the stress varies $\approx 10 \%$ from its maximum value over the Knudsen layer thickness of $2 \lambda$ ).

Using Eq. (A1) for velocity slip, the Navier-Stokes solution for the mass-flow rate $m$ is

$$
m=-\frac{2 \rho G h^{3}}{3 \mu}(1+3 A \mathrm{Kn}),
$$

where $\rho$ is the density, $\mu$ is the viscosity, $G$ is the applied pressure gradient, and $h$ is half the height of the channel. At $\mathrm{Kn}=0.05$, the percentage increase in mass-flow rate due to slip and Knudsen layer effects is therefore $\approx 17 \%$.

According to Cercignani's solution, the actual (or "micro" slip) can be obtained with a slip coefficient $A=\sqrt{2 / \pi}$ $\approx 0.8$. By substituting this value into Eq. (A2) we can evaluate the contribution to the increased mass-flow rate that is solely due to velocity slip. This contribution is $\approx 70 \%$ of the change in mass-flow rate. The remaining $30 \%$ is due to the non-Newtonian structure of the Knudsen layer.

\section{APPENDIX B}

The Knudsen layer is characterized by a nonlinear relationship between stress and rate of strain, but it is a linear phenomenon, i.e., it does not rely on the presence of strong flow gradients to exist. This is supported theoretically by linear kinetic theory ${ }^{2,3}$ and the low-shear DSMC simulations of this paper. The nonlinear terms featuring in higher-order continuum equations, which are products of spatial derivatives, can therefore justifiably be neglected in the study of a low-speed isothermal Knudsen layer.

To verify this assertion we have used DSMC data to evaluate the magnitude of the possible contribution to shear stress within the Knudsen layer from higher-order nonlinear terms. For the Burnett, super-Burnett, and Grad equations (up to third order in Knudsen number) the only nonlinear term that exists for the one-dimensional isothermal Knudsen layer is of the form:

$$
\frac{\mu^{3}}{p^{2}}\left(\frac{d \nu}{d x}\right)^{3}
$$

Using the DSMC data shown in Figs. 2 and 3, the magnitude of this term has been evaluated at various points in the Knudsen layer. The nonlinear contribution to the overall stress is less than $0.05 \%$ in all cases, demonstrating that the linearization of the higher-order constitutive relations for Kramer's problem is appropriate.

${ }^{1}$ M. Gad-el-Hak, "Comments on "critical view on new results in micro-fluid mechanics'," Int. J. Heat Mass Transfer 46, 3941 (2003).

${ }^{2}$ M. N. Kogan, Rarefied Gas Dynamics (Plenum, New York, 1969), pp. 367-399.

${ }^{3}$ C. Cercignani, Mathematical Methods in Kinetic Theory (Plenum, New York, 1990), pp. 232-243.

${ }^{4}$ Y. Sone, Kinetic Theory and Fluid Dynamics (Birkhauser, Boston, 2002).

${ }^{5}$ G. A. Bird, Molecular Gas Dynamics and the Direct Simulation of Gas Flows (Clarendon, Oxford, 1994).

${ }^{6} \mathrm{~S}$. Chapman and T. G. Cowling, The Mathematical Theory of NonUniform Gases (Cambridge University Press, Cambridge, 1970).

${ }^{7}$ C. S. Wang-Chang, University of Michigan Department of Engineering Report No. UMH-3-F(APL.JHU CH-467), 1948.

${ }^{8} \mathrm{H}$. Grad, "On the kinetic theory of rarefied gases," Commun. Pure Appl. Math. 2, 331 (1949).

${ }^{9}$ R.-S. Myong, "Thermodynamically consistent hydrodynamic computational models for high-Knudsen-number gas flows," Phys. Fluids 11, 2788 (1999).

${ }^{10}$ X. Zhong, R. W. MacCormack, and D. R. Chapman, "Stabilization of the Burnett equations and application to hypersonic flows," AIAA J. 31, 1036 (1993).

${ }^{11}$ F. E. Lumpkin III, "Development and evaluation of continuum models for translational-rotational nonequilibrium," Ph.D. thesis, Department of Aeronautics and Astronautics, Stanford University, 1990.

${ }^{12} \mathrm{R}$. Balakrishnan, "An approach to entropy consistency in second-order hydrodynamic equations," J. Fluid Mech. 503, 201 (2004).

${ }^{13} \mathrm{~S}$. Jin and M. Slemrod, "Regularization of the Burnett equations via relaxation,” J. Stat. Phys. 103, 1009 (2001).

${ }^{14} \mathrm{H}$. Struchtrup and M. Torrilhon, "Regularization of Grad's 13 moment equations: derivation and linear analyis," Phys. Fluids 15, 2668 (2003).

${ }^{15}$ L. C. Woods, "Frame-indifferent kinetic theory," J. Fluid Mech. 136, 423 (1983).

${ }^{16}$ D. A. Lockerby, J. M. Reese, and M. A. Gallis, "A wall-function approach to incorporating Knudsen-layer effects in gas micro flow simulations," Proceedings of the 24th International Symposium on Rarefied Gas Dynamics, Bari, 2004. 